\title{
Early Deficits in Spatial Memory and Theta Rhythm in Experimental Temporal Lobe Epilepsy
}

\author{
Laetitia Chauvière, ${ }^{1}$ Nadia Rafrafi, ${ }^{1}$ Catherine Thinus-Blanc, ${ }^{1}$ Fabrice Bartolomei, ${ }^{1,2}$ Monique Esclapez, ${ }^{1}$ \\ and Christophe Bernard ${ }^{1}$ \\ ${ }^{1}$ Inserm-U751, Université de la Méditerranée, 13005 Marseille, France, and ²Assistance Publique des Hôpitaux de Marseille Hôpital La Timone, 13005 \\ Marseille, France
}

\begin{abstract}
Patients with temporal lobe epilepsy (TLE), the most common form of epilepsy in adults, often display cognitive deficits. The time course and underlying mechanisms of cognitive decline remain unknown during epileptogenesis (the process leading to epilepsy). Using the rat pilocarpine model of TLE, we performed a longitudinal study to assess spatial and nonspatial cognitive performance during epileptogenesis. In parallel, we monitored interictal-like activity (ILA) in the hippocampal CA1 region, as well as theta oscillations, a brain rhythm central to numerous cognitive processes. Here, we report that spatial memory was altered soon after pilocarpine-induced status epilepticus, i.e., already during the seizure-free, latent period. Spatial deficits correlated with a decrease in the power of theta oscillations but not with the frequency of ILA. Spatial deficits persisted when animals had spontaneous seizures (chronic stage) without further modification. In contrast, nonspatial memory performances remained unaffected throughout. We conclude that the reorganization of hippocampal circuitry that immediately follows the initial insult can affect theta oscillation mechanisms, in turn, resulting in deficits in hippocampusdependent memory tasks. These deficits may be dissociated from the process that leads to epilepsy itself but could instead constitute, as ILA, early markers in at-risk patients and/or provide beneficial therapeutic targets.
\end{abstract}

\section{Introduction}

Cognitive deficits remain a major complaint in temporal lobe epilepsy (TLE), the most common form of partial epilepsy in adults (Engel et al., 2007). The origin of cognitive comorbidity remains widely unknown. Many contributing factors have been proposed (Cornaggia et al., 2006), including seizures themselves (Aldenkamp and Arends, 2004; Majak and Pitkanen, 2004; Marques et al., 2007), the abnormal electrical activities that occur between seizures (Aldenkamp and Arends, 2004), antiepileptic drugs (Meador, 2006), and the reorganization of the underlying neuronal circuitry (Morimoto et al., 2004). Seizures and the interictal activity (IA) that consistently occurs between seizures could negatively impact upon cognitive function, via saturation of long-term synaptic plasticity and interference with memory consolidation (Lopes da Silva et al., 1986; Kotloski et al., 2002; Aldenkamp and Arends, 2004; Majak and Pitkanen, 2004; Holmes and Lenck-Santini, 2006; Meador, 2007; Butler and Zeman, 2008).

Experimental models of TLE display a seizure-free (latent) period after an initial insult (Herman, 2002; White, 2002; Engel et

Received Sept. 30, 2008; revised Feb. 23, 2009; accepted Feb. 27, 2009.

This work was supported by Inserm and Agence Nationale de la Recherche ANTARES. L.C. has a Fondation pour la Recherche Medicale fellowship. We thank C. Silva and M.-P. Nesa for assistance with histological experiments, Astrid Nehlig for training L.C. with depth EEG recordings, M. Milh for making the first measurements of theta activity during epileptogenesis, and D. Turner and C. Flynn for critically reading this manuscript.

Correspondence should be addressed to Laetitia Chauvière, Inserm-U751, Université de la Méditerranée, 27, Boulevard Jean Moulin, 13005 Marseille, France. E-mail: laetitia.chauviere@univmed.fr.

C. Thinus-Blanc's present address: UMR 6146, CNRS, Université de Provence, 3, place Victor Hugo, 13331 Marseille, France.

DOI:10.1523/JNEUROSCI.4699-08.2009

Copyright $\odot 2009$ Society for Neuroscience $\quad$ 0270-6474/09/295402-09\$15.00/0 al., 2007). The initial insult triggers a chain of network alterations which evolve temporally and spatially during epileptogenesis (Herman, 2002; El-Hassar et al., 2007). Hippocampal networks are hyperexcitable already during the latent period (El-Hassar et al., 2007), and EEG displays interictal-like activity (ILA), similar to the IA measured between seizures (Staley et al., 2005; El-Hassar et al., 2007). Classical tests demonstrate spatial memory deficits already during the latent period (Rice et al., 1998; Hort et al., 1999; Dos Santos et al., 2005). The first goal of this study was to determine the time course of cognitive deficits during epileptogenesis. We also wished to determine whether nonspatial hippocampal-independent memory was preserved to verify the specificity of cognitive alterations. For that purpose, we used a modification of the "novel object exploration" test (Berlyne, 1950). When a feature of a previously explored environment is modified, animals display a renewal of exploratory activity selectively directed toward the unfamiliar feature. In this test, previously stored spatial and nonspatial information serves as a reference for novelty detection. Rats with hippocampal lesions display spatial, but not nonspatial, memory deficits (Save et al., 1992a). This paradigm is thus suitable to dissociate treatments which selectively affect spatial or nonspatial memory.

The second goal of this study was to correlate cognitive alterations with ILA and theta rhythm $(4-12 \mathrm{~Hz})$, which plays a key role in cognitive processes in both humans (Kahana et al., 1999) and rodents (Buzsaki, 2006). Since decreased and increased theta activity correlate with cognitive deficit and improvement, respectively (Kinney et al., 1999; Nolan et al., 2004; McNaughton et al., 2006), compromised theta activity during epileptogenesis could be an important contributing factor for cognitive dysfunction. 
Using the pilocarpine model of TLE, we report an early and persistent deficit of spatial memory during epileptogenesis, whereas nonspatial memory remained unaffected. Decreased theta activity in the hippocampus correlated with cognitive deterioration, whereas interictal activity did not. We suggest that cognitive alterations may result from a decreased ability to generate adequate theta activity and that both alterations may constitute early markers of epileptogenesis in at-risk patients.

\section{Materials and Methods}

Animals. All tests were performed during the light phase of the cycle on 18 adult male Wistar rats (200-250 g; Charles River).

Electrode implantation. Rats were anesthetized with ketamine $(1 \mathrm{mg} /$ $\mathrm{kg}) /$ xylasine $(0.5 \mathrm{mg} / \mathrm{kg})$ and placed in a stereotaxic apparatus. One bipolar steel electrode was implanted in the dorsal hippocampus $(3.8 \mathrm{~mm}$ anteroposterior, $1.5 \mathrm{~mm}$ mediolaterally, and $3.0 \mathrm{~mm}$ dorsoventral from the bregma). Three stainless-steel cortical electrodes were screwed on the skull (two screws in the right and left frontal cortex, and the reference in the cerebellum).

Treatment. Twenty-five rats received intraperitoneal injections of pilocarpine hydrochloride $\left(310 \mathrm{mg} \mathrm{kg}^{-1}\right), 30 \mathrm{~min}$ after an intraperitoneal injection of scopolamine injection $(1 \mathrm{mg} / \mathrm{kg}$ ). Status epilepticus (SE) was stopped by diazepam $(8 \mathrm{mg} / \mathrm{kg})$ after $40 \mathrm{~min}$. Eight animals displayed stage 5 seizures but did not develop SE (non-SE group). These animals did not become epileptic within 3 months, and their behavioral, cognitive, and EEG activities remained intact. They were included in the control group, which also included two sham-treated rats, which received saline injection instead of pilocarpine. All drugs were obtained from Sigma.

In vivo depth EEG recordings. Recordings were performed before ( $1 \mathrm{~h})$, during, and after $(1 \mathrm{~h})$ the task (Deltamed). The same protocol was performed 4, 7, 10, 25, and $40 \mathrm{~d}$ after treatment for each rat. A videosystem (Video Track; View Point) was used to quantify behavior during recording sessions. The first spontaneous seizure occurred between 12 and $16 \mathrm{~d}$ after SE. The 4,7 , and $10 \mathrm{~d}$ post-SE corresponds to latent period time points. At 25 and $40 \mathrm{~d}$, animals were epileptic (chronic period). It is important to note that EEG monitoring during the induction procedure is critical. We noted that electrographic status epilepticus (continuous electrographic seizure, SE) started as soon as the first overt behavioral manifestation. After diazepam injection, most animals stopped any behavioral manifestation, but in $50 \%$ of the cases, electrographic and/or behavioral SE did not stop. We have performed continuous 24/24 h 7/7 d recordings after pilocarpine injection (seven animals). We noted that two animals displayed a strong SE after pilocarpine injection. By strong SE, we mean many stage 5 seizures before the development of SE and a strong behavioral activity during the SE. These animals displayed another seizure within $12 \mathrm{~h}$ (usually during the night). No other seizures were observed till the beginning of the chronic period. All animals that experienced SE after pilocarpine injection developed spontaneous seizures, which were electrophysiologically recorded and/or observed behaviorally $(n=19)$.

Behavioral testing. An open field ( $80 \mathrm{~cm}$ in diameter, $80 \mathrm{~cm}$ high) was used, with a yellow cue card affixed on the wall. Lighting was directed to the ceiling to provide a homogeneous dim illumination of the open field. Before the task, the rat was put in the open field for $1 \mathrm{~h}$ to allow it to explore, first, the novel environment without any object. Exploration is crucial for spatial learning and considered as a necessary stage for gathering information of the surrounding environment and for building up a spatial representation of this new environment (Thinus-Blanc, 1996). The rat was then removed and replaced in its home cage during $10 \mathrm{~min}$. The task consisted of three sessions. In the first session, two objects (a cylinder and a parallelepiped) were placed in the open field. The parallelepiped ( $10 \mathrm{~cm}$ of side, $7.0 \mathrm{~cm}$ of height) was considered as the reference object. The other object was a cylinder $(7.0 \mathrm{~cm}$ of diameter and $7.0 \mathrm{~cm}$ of height). In the second session, the cylinder was displaced. In the third session, this object was removed and replaced by a triangular prism $(7.0$ $\mathrm{cm}$ of height, $14.0 \times 10.0 \mathrm{~cm}$ of side). All three objects were made of light gray-colored plastic with a black top, and they were heavy enough to prevent rats from moving them. They could not be distinguished by olfactory cues. Pilot studies (our unpublished data) showed that there were no preferences for either of the objects, or for their location in the open field. Each session consisted in five 2 min trials. Rats were placed in the field in pseudo-randomly determined locations from one session to the other. Between each trial and each session, there were 2 min of rest during which the rat was replaced in its home cage. Exploration of the objects was quantified by the Video Track system, which detects the nose of the animal in $2 \mathrm{~cm}$ zones around the objects.

Data analysis. Power spectrum was analyzed using a 512-point fast Fourier transform. For each exploration period, we obtained the absolute power and the median frequency of hippocampal theta rhythm (4-12 $\mathrm{Hz}$ ) every $2 \mathrm{~s}$ (AMADEUS software). We used $200 \times 2 \mathrm{~s}$ exploration periods for each rat. The movement artifacts and the periods of ILA discharges were manually removed of the analysis. The hippocampal EEG trace was filtered with a bandpass filter at $4-12 \mathrm{~Hz}$ to obtain the median frequency of theta rhythm each $2 \mathrm{~s}$.

Statistical analysis. Behavioral data were analyzed using the Wilcoxon test, a nonparametric test that compares two paired samples (for the data before treatment, and for comparisons between the exploration of two objects for each group after treatment, for the three sessions), the unpaired Mann-Whitney test (for comparisons between the two groups, after treatment, for the three sessions), and the paired Friedman test (to measure the performances stability for each group all along the six testing days). We also used the Kruskal-Wallis test, a nonparametric test that compares many unpaired samples [for comparisons between the two groups for the first session (S1), all along the five trials]. The absolute power and frequency of theta rhythm was compared between experimental and control rats using a Student's $t$ test for unpaired samples and a one-way ANOVA followed by Bonferroni's post hoc test. Spearman correlation was used to measure the correlation between cognition and theta rhythm power and between cognition and interictal-like activity. Data are expressed as mean \pm SEM. The significance level was set at $p<$ 0.05. Statistical analysis was performed using SPSS software.

Histological experiments. At the end of the electrophysiological experiments, 7-40 d after injections, all the rats were deeply anesthetized with ketamine $(1 \mathrm{mg} / \mathrm{kg}) /$ xylasine $(0.5 \mathrm{mg} / \mathrm{kg})$ and perfused intracardially with a fixative solution containing $4 \%$ paraformaldehyde in $0.12 \mathrm{M} \mathrm{so-}$ dium phosphate buffer ( $\mathrm{PB}), \mathrm{pH}$ 7.4. After perfusion, the brains were postfixed in the same fixative for $1 \mathrm{~h}$ at room temperature (RT), rinsed in $0.12 \mathrm{M}$ PB for $1.5 \mathrm{~h}$, immersed in a cryoprotective solution of $20 \%$ sucrose in $\mathrm{PB}$ overnight at $4^{\circ} \mathrm{C}$, quickly frozen on dry ice, and sectioned coronally at $40 \mu \mathrm{m}$ with a cryostat. Every tenth section was stained with cresyl violet to determine the general histological characteristics of the tissue within the rostral-caudal extent of the hippocampal formation including the entorhinal cortex, as well as to verify electrode placement. Its position within the CA1 region of the hippocampus was confirmed in all reported animals (supplemental Fig. S4, available at www.jneurosci.org as supplemental material).

In addition, to conduct a more detailed quantitative stereological study of neuronal populations within the hippocampus, dentate gyrus, and medial entorhinal cortex, immunohistochemical experiments for the detection of neuronal-specific nuclear protein (NeuN) were performed on sections selected at regular intervals within the rostrocaudal extend of these structures (see below).

Immunohistochemical methods. Free-floating sections were first rinsed for $30 \mathrm{~min}$ in $0.02 \mathrm{M}$ potassium PBS (KPBS; $16 \mathrm{~mm} \mathrm{~K}_{2} \mathrm{HPO}_{4}, 3.5 \mathrm{~mm}$ $\mathrm{KH}_{2} \mathrm{PO}_{4}, 150 \mathrm{~mm} \mathrm{NaCl}, \mathrm{pH} 7.4$ ), incubated for $30 \mathrm{~min}$ in $1 \%$ hydrogen peroxide to block endogenous peroxidases, and rinsed in KPBS. Sections were then incubated for $1 \mathrm{~h}$ at RT in KPBS containing 0.3\% Triton X-100 (KPBS-Triton) and 3\% normal horse serum (NHS; Vector Laboratories), and overnight at RT in NeuN monoclonal antibody (MAB 377; Millipore Bioscience Research Reagents) diluted 1:4000 in KPBS-Triton containing 1\% NHS. After these steps, sections were rinsed for $30 \mathrm{~min}$ in KPBS and incubated for $1 \mathrm{~h}$ at RT in biotinylated horse anti-mouse IgG (Vector Laboratories) diluted 1:200 in KPBS containing 3\% NHS. Sections were then rinsed in KPBS, incubated for $1 \mathrm{~h}$ at RT with an avidinbiotin-peroxidase complex solution prepared according to the manufacturer's recommendations (Vector Laboratories). After several rinses in 
KPBS, sections from control and pilocarpinetreated animals were processed for the same time (10 $\mathrm{min}$ ) in $0.04 \%$ 3-3-diaminobenzidine- $\mathrm{HCl}$ and $0.006 \%$ hydrogen peroxide diluted in KPBS. The sections were then rinsed in KPBS, mounted on gelatin-coated slides, dried, dehydrated, and coverslipped with permount (Fisher Scientific; Electron Microscopy Sciences).

Quantitative analysis. In this study, we estimated the total numbers of NeuN-labeled neurons in the CA1 and CA3 pyramidal cell layers, as well as in layers I-II, III, and V-VI of the medial entorhinal cortex, in one cerebral hemisphere of control and pilocarpine-treated animals, by using the optical fractionator method (West et al., 1991; West, 1999). These analyses were performed with a computer-assisted system using the Stereo Investigator software (MicroBrightField Bioscience). The computerassisted system included a PC connected to a color video camera (CX 9000; MicroBrightField Bioscience) connected to a Nikon microscope (E90I; Nikon). The neurons were counted with a $60 \times$ objective. For the hippocampus, starting from a random position, the number of sections analyzed was 12 and 6 for the entorhinal cortex. In each section, the CA1 and CA3 pyramidal cell layers were delineated as described in previous stereological studies (West et al., 1991; Dinocourt et al., 2003). The borders of the medial entorhinal cortex were identified according to Mulders et al. (1997) and Paxinos and Watson (2005). Neurons were counted within a probe volume defined by the counting frame and the dissector height. The dissector height $(20 \mu \mathrm{m})$ used in this study is the real thickness of sections $(24 \mu \mathrm{m})$ minus $2 \mu \mathrm{m}$ on each side of the section to exclude the regions showing irregularities because of cut processes. The real thickness of sections $(24 \mu \mathrm{m})$ resulted from shrinkage of the sections $(40 \mu \mathrm{m}$ thick) after histological treatment. The size of the counting frame was $50 \times 50 \mu \mathrm{m}$ and of the counting grid was $400 \times 400 \mu \mathrm{m}$, for the CA1 and CA3 pyramidal cell layers. The size of the counting frame was $50 \times 50 \mu \mathrm{m}$ and of the counting grid was $300 \times 300 \mu \mathrm{m}$ for the different layers of the medial entorhinal cortex. Only neurons within the counting frame or overlapping the right or superior border of the counting frame, and which came into focus while focusing down through the dissector height, were counted. The average number of neurons counted per layer in each animal is indicated in supplemental Table S6, available at www.jneurosci.org as supplemental material. The precision of each estimate was controlled by measuring the coefficient of error (CE), which is the mean of the coefficients of error of the individual estimates (West et al., 1991; West, 1999). The CE was $10 \%$ or less for each estimated number of labeled neurons, and the mean $\mathrm{CE}$ is indicated in supplemental Table $\mathrm{S6}$, available at www.jneurosci.org as supplemental material. The total numbers of neurons labeled per layer and per cerebral hemisphere for each animal were estimated by the software. The total number of neurons in all layers of the medial entorhinal cortex was obtained by summing the number of cells in layers I-II, III, and V-VI for each animal. The average estimated total number of labeled neurons \pm SEM was calculated for each group of control and pilocarpine-treated rats (supplemental Table S6, available at www.jneurosci.org as supplemental material). The data were analyzed statistically with a mixed-model ANOVA and Student's $t$ test to determine significant differences in the number of neurons per layer between groups (control and pilocarpine-treated rats). The extent of neuron loss in pilocarpine-treated rats was calculated with the following formula: [100 - $(100 \times$ average estimated number of neurons in pilocarpine-treated rats/average estimated number of neurons in control rats) $] \%$.

\section{Results}

Spatial memory impairment during epileptogenesis

The reaction-to-a-change task (Fig. 1) was performed to assess cognitive abilities for hippocampus-dependent spatial and
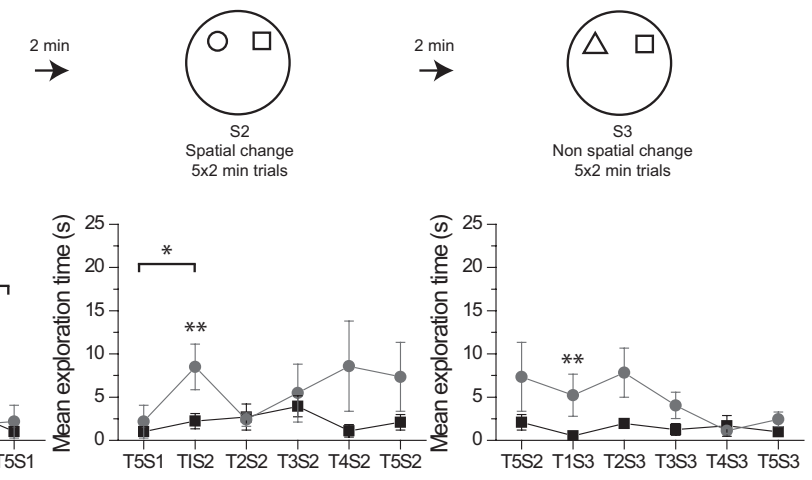

Figure 1. Spatial and nonspatial performances before treatment. Top, Experimental paradigm. During the S1 session, animals explore the two new objects ( 5 trials of 2 min each). One object is moved to a different quadrant, and animals are allowed to explore ( $\$ 2$ session, 5 trials of 2 min each). One object is then replaced ( $\$ 3$ session, 5 trials of 2 min each). Bottom, Mean duration (sec.) spent exploring the familiar object (black squares) and the other object (red circles) during session 1 (left histogram), session 2 (central histogram), and session 3 (right histogram). There was a significant habituation for both objects between the first and the last (T5S1) trial ( $p<0.0001 ; n=18)$. Object displacement led to renewed exploration, as compared with the time spent exploct in the last trial of S1 (T5S1; T1S2/T5S1: $p=0.009, n=18)$. Object replacement led to renewed sma significantly smaller than the time spent exploring the new object, showing that animals had detected the novelty ( $p=0.049$ $n=18$ ). Data are expressed as means \pm SEM and ${ }^{*} p<0.05$.

hippocampus-independent nonspatial memories (ThinusBlanc, 1996), 7 d before injection (D7). Each animal thus served as its own control in this longitudinal study. Rats $(n=18)$ were initially placed in an open field containing two different objects and were allowed to explore (S1). Exploration quickly diminished across the five trials (Fig. 1) $(p<0.0001)$, as animals habituated to the objects. After habituation, one object was displaced (second session, S2), leading to increased exploration of the displaced object (Fig. 1), as compared with the exploration of this object during the last trial of S1 [trial 1 (T1)S2: mean duration of displaced object exploration: $8.5 \pm 2.6 \mathrm{~s}, p<0.05$ vs T5S1: $1.0 \pm 0.6 \mathrm{~s}$ ) and to the exploration of the reference object (Fig. 1) (T1S2 of reference object: $2.2 \pm 0.9 \mathrm{~s}, p<0.05$ ), which remained at the same level as at the end of session S1. After the fifth trial of the S2 session, one object was changed (third session, S3). There was a trend toward increased exploration of the new object compared with the exploration of the displaced object in the last trial of S2 (T5S2), but it failed to reach significance (T1S3: mean duration of new object exploration: $5.3 \pm 2.4 \mathrm{~s}, p=0.225$ vs T5S2) (Fig. 1) because of the reaction to the spatial change persisted across the five trials of the S2 session. However, there remained a preference for novelty, since the time spent exploring, in T1S3, the new object was greater than the time spent exploring the familiar object (new object, $5.2 \pm 2.4 \mathrm{~s}$; familiar object, $0.5 \pm 0.3$; $p<0.05)$.

After 1 week rest (D0), animals received either saline $(n=2)$ or pilocarpine $(n=16)$. Animals that did not exhibit pilocarpineinduced SE but did show evidence of generalized (stage 5) seizures after treatment $(n=4)$ were included in the control group. Cognitive performance was then evaluated 4, 7, 10, 25, and $40 \mathrm{~d}$ after the injection (D4, D7, D10, D25, and D40). Experimental animals that exhibited SE $(n=12)$ did not show any habituation of exploratory activity between the first trial (T1S1) and the fifth trial (T5S1) of S1. This result was found as early as D4 and persisted without further modification during epileptogenesis (Fig. $2 A$; supplemental Table S1, available at www.jneurosci.org as supplemental material) ( $p=0.250)$. The control group still displayed habituation between T1S1 and T5S1 at D4, D7, and D25. 
A
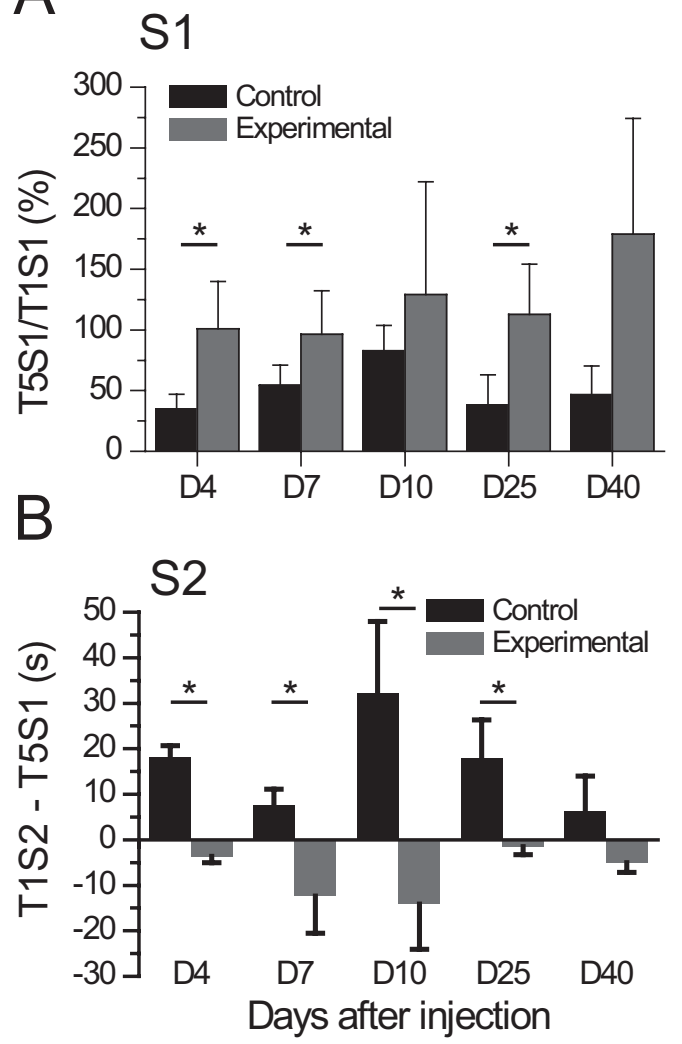

C

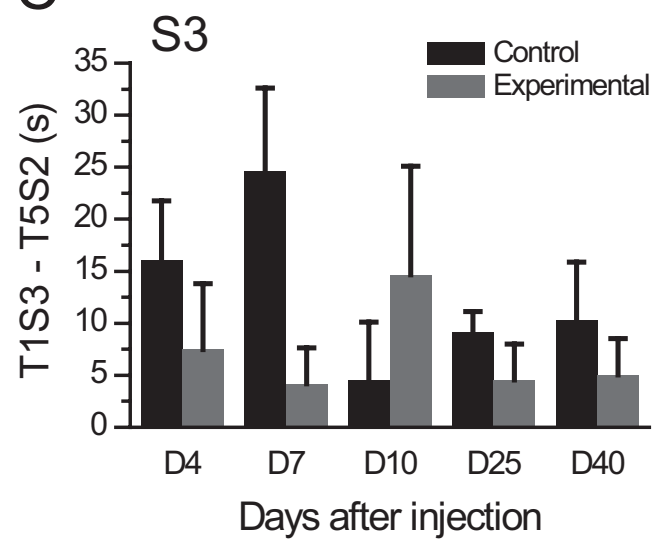

Figure 2. Early and persistent deficit in spatial memory task in experimental TLE. A, Ratio of the time spent exploring both objects during T5S1 versus the time spent exploring both objects during T1S1, during the latent and chronic periods. A ratio $<100 \%$ indicates less exploration, i.e., habituation. Experimental animals $(n=12)$ did not present any habituation of exploration of the two objects, between the first and the fifth trial for all testing days after SE (D4, D7, D25, and D40; $p>0.05)$ compared with the control group $(n=6)$, except at D10 and D40, where there was instead a significant decrease of exploration between T3S1 and T5S1 for the control group, caused by a delayed reaction to the objects. There was no significant effect ( $p>0.05$ ) all along the five testing days, for both groups. $\boldsymbol{B}$, Difference between the time (s) spent exploring the displaced object in T1S2 and the time (s) spent exploring the same object in T5S1 (T1S2-T5S1) 4, 7, 10, 25, and 40 d after injection. Positive values indicate renewed exploration, hence spatial memory function (control/experimental, $\left.{ }^{*} p<0.05\right)$. Experimental animals $(n=12)$ did not present any significant interest for the spatial change as compared with controls animals $(n=6$; experimental/control, $p<0.05)$. There was no significant effect $(p>0.05)$ all along the five testing days, for both groups. C, Difference between the time (s) spent exploring the new object in T1S3 and the time (s) spent exploring the former object in T5S2 (T1S3-T5S2) 4, 7, 10, 25, and $40 \mathrm{~d}$ after injection. Positive values indicate renewed exploration, hence nonspatial memory function $\left({ }^{*} p<0.05\right)$. Experimental ( $n=12)$ and control $(n=6)$ animals displayed a significant interest to novelty (control/ experimental, $p>0.05)$. Therewas no significant effect $(p>0.05)$ all along the fivetesting days, for both groups. Data are expressed as means \pm SEM.
However, at D10 and D40, there was instead a significant decrease of exploration between T3S1 and T5S1 for control animals, caused by a delayed reaction to the objects $(n=6)$ (Fig. $2 A$; supplemental Table S1, available at www.jneurosci.org as supplemental material) $(p<0.05)$.

Experimental animals did not show any preference for the displaced object in S2, already at D4, indicating a deficit of spatial memory. In contrast, the control group showed the normal increased exploration of the displaced object (Fig. $2 B$; supplemental Table S2, available at www.jneurosci.org as supplemental material) (control/experimental, $p<0.0001$ at D4). The deficit of spatial memory persisted at all later time points in the experimental group (Fig. $2 B$; supplemental Table S2, available at www. jneurosci.org as supplemental material $)(p<0.05)$, except at D40, where a trend was found between the two groups ( $p=$ $0.06)$. Spatial memory performance remained stable in the control group (Fig. $2 B$; supplemental Table S2, available at www. jneurosci.org as supplemental material). The deficit in the reaction to the spatial change in experimental animals was not attributable to a simple deficit in motor or exploratory activities, since animals did explore objects (supplemental Table S1, supplemental Fig. S1, available at www.jneurosci.org as supplemental material). There is, however, a tendency for experimental rats to explore less than the control group; this tendency becoming significant only at D25 and D40 (Fig. 2 A; supplemental Table S1, available at www.jneurosci.org as supplemental material) $(p<$ $0.05)$.

In contrast to the spatial memory task, experimental animals displayed an interest for the nonspatial change ( $p<0.05$ at D4, D7, and D25) (Fig. 2C), which was indistinguishable from the control group at all time points (Fig. $2 C$; supplemental Table S3, available at www.jneurosci.org as supplemental material) $(p=$ 0.399 ). This suggests that hippocampus-independent nonspatial memory is not affected in experimental TLE.

To ascertain that the relatively low level of object exploration of the experimental animals was not attributable to anxiety (i.e., to a preference for the periphery of the open field), the pathways of the animals recorded by the Videotrack system were visually inspected. None of the experimental animals moved exclusively around the borders of the field; instead, they crossed the center of the field several times (supplemental Fig. S1, available at www. jneurosci.org as supplemental material). If some anxiety-related behavior cannot be excluded on the basis of the lower level of exploration, the fact that the experimental rats did react to the nonspatial change allows us to discard the possibility that their lack of reaction to the spatial change was exclusively related to anxiety.

To rule out the possibility that our initial group assignment procedure could have biased our results, we retrospectively examined the initial cognitive performance of both the experimental and control groups. There was no statistical difference for habituation or reaction-to-a-change between both groups (S2, $p=0.573 ; \mathrm{S} 3, p=0.759$ ). Therefore, the initial cognitive performances were similar for all animals before treatment.

Finally, there was no statistical difference for the cognitive performances of the two groups across the six testing days after saline or pilocarpine treatment: cognitive processing of both spatial and nonspatial tasks was stable in the control group (S2, $p=$ 0.282 ; $S 3, p=0.491$ ), and nonspatial performance was not affected during epileptogenesis in experimental animals $(p=$ 0.896). Moreover, the repetition of the task at the different testing days did not affect a global level decrease of exploratory activity (Fig. 2A,B). 

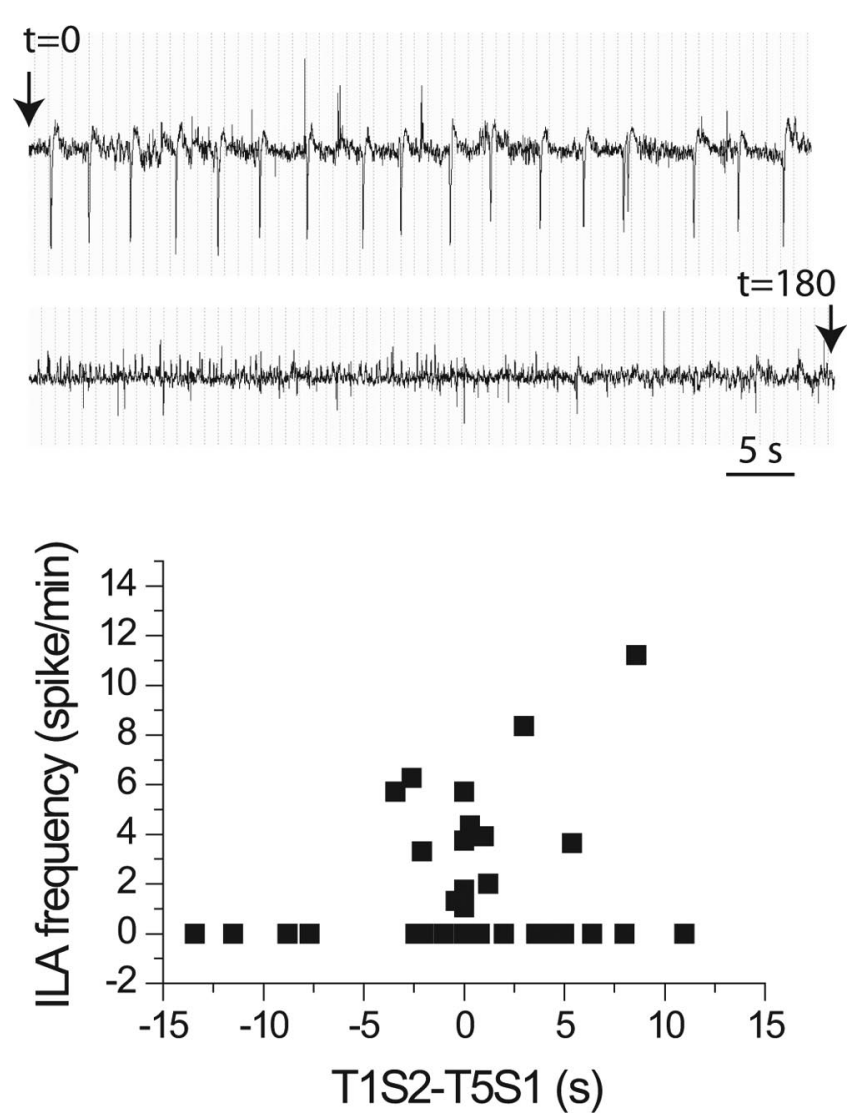

Figure 3. Lack of correlation between ILA and spatial deficit in experimental animals ( $n=$ 25). Top, Typical burst of ILA lasting 3 min recorded at D10 during rest. Note that the pattern evolves from large amplitude and slow to shorter amplitude and faster spike and waves. Bottom, There was no correlation between ILA frequency and spatial cognitive deficit measured as the T1S2-T5S1 difference $(r=0.015 ; p=0.913)$.

These findings indicate that the deficit of spatial processing is an early and persistent phenomenon in experimental animals and that nonspatial processing is not affected for the cognitive tasks considered here.

\section{Lack of correlation between cognitive deficits and interictal activity}

Extracellular field recordings revealed that ILA appeared in bursts in the hippocampus $7 \mathrm{~d}$ after SE in all animals, except for one rat, which displayed ILA at D4. Spatial cognitive deficits did not correlate with the mean frequency (number of spike-andwaves per minute, in $1 \mathrm{~h}$ ) of ILA measured before the task at D7, D10, and D25 (Fig. 3) (Spearman correlation; $p=0.9$ ).

\section{Cognitive deficits correlate to decreased theta activity during epileptogenesis}

Experimental animals displayed decreased absolute power and median frequency of theta rhythm at all time points when compared with the control group during exploratory activity in the open field, $1 \mathrm{~h}$ before and after the task (Fig. $4 A-C$; supplemental Table S4, available at www.jneurosci.org as supplemental material) ( $p<0.0001$ for both power and frequency). During the task, the absolute power of theta rhythm was also decreased in experimental animals at all time points (Fig. $4 D$ ) in S1 (habituation of exploratory activity; $p<0.0001$ ) and S2 (spatial change; $p<$ $0.0001)$. In contrast to exploration in the open field in the absence of objects, theta frequency was variable during the S1, S2, and S3 tasks between rats in both sham and experimental groups, precluding direct comparisons.

Spearman correlation between the mean duration of objectdisplaced exploration and the absolute theta power during T1S2 at all time points revealed a significant positive correlation between the decrease in theta power and the cognitive deficits in experimental animals (Fig. $4 E)(r=0.5 ; p=0.00002)$. In contrast, there was no correlation between the mean duration of new object exploration and the absolute theta power during T1S3 (data not shown; $p=0.263$ ).

Global EEG power spectrum $(0-100 \mathrm{~Hz})$ as well as gamma rhythm $(40-80 \mathrm{~Hz})$ measured before and after the task remained stable in both experimental and control groups at all time points (supplemental Fig. S2, available at www.jneurosci.org as supplemental material). The decrease of theta power in experimental rats thus appears to be particular to this bandwidth.

Since speed of movement affects theta power (Maurer et al., 2005), we evaluated this parameter in sham and experimental animals. There was no speed difference between the experimental and the control groups (supplemental Fig. S3, available at www.jneurosci.org as supplemental material), suggesting that theta decrease is not attributable to a loss of activity of experimental rats, or to a general sick state of the animal (see also supplemental Fig. S1, available at www.jneurosci.org as supplemental material).

\section{Pattern of cell loss in experimental animals}

Decreased theta power in experimental animals could result from a lesion attributable to the recording electrode and/or excitotoxic damage induced by SE. Histological analysis revealed a lack of marked damage caused by the recording electrode (supplemental Fig. S4, available at www.jneurosci.org as supplemental material) and of a major cell loss in this model. The pattern of neuronal damage observed in the hippocampus and dentate gyrus in pilocarpine-treated animals after a 40-min-long SE was very similar to that described previously in pilocarpine-treated animals after 2-3-h-long SE (Obenaus et al., 1993; Esclapez et al., 1999; Dinocourt et al., 2003; Boulland et al., 2007). It included a cell loss restricted to the hilus of the dentate gyrus and stratum oriens of CA1 that contrasts with a good preservation of neurons in all other layers of the hippocampus and dentate gyrus including the CA1-CA3 pyramidal cells and dentate granule cell layers (supplemental Fig. S5, available at www.jneurosci.org as supplemental material). No obvious cell loss was observed in the medial entorhinal cortex (supplemental Fig. S6, available at www.jneurosci.org as supplemental material), in contrast to the previously reported major cell loss described in animals that experienced longer-lasting SE (Clifford et al., 1987; Du et al., 1995; Kumar and Buckmaster, 2006).

Because, in the pilocarpine model, partial sclerosis was described in the hippocampal pyramidal cell layers (Liu et al., 1994) as well as in layers II and III of the medial entorhinal cortex (Du et al., 1995; Kumar and Buckmaster, 2006), we conducted a quantitative stereological analysis of the total number of neurons in these regions to estimate eventual subtle cell loss. This analysis revealed a slight neuronal loss in all CA1-CA3 pyramidal cell layers of pilocarpine-treated rats compared with control animals. In CA1, the estimated total number of neurons was reduced by $10 \%$ as compared with control [pilocarpine (pilo), $303360 \pm$ 7039; control, $337920 \pm 3866 ; p<0.01$ ], whereas in CA3 the estimated total number of neurons was reduced by $13 \%$ (pilo, $207360 \pm 4796$; control, $238080 \pm 3104 ; p<0.005$ ) (supplemental Table S5, available at www.jneurosci.org as supplemental material; Fig. $5 A$ ). In the medial entorhinal cortex, the quantitative 
A

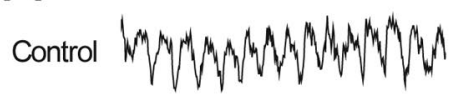

B

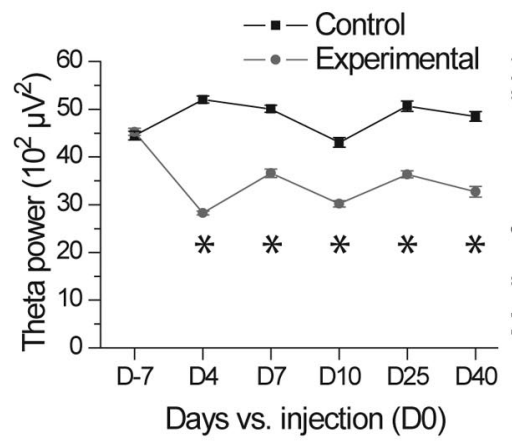

\section{During test}

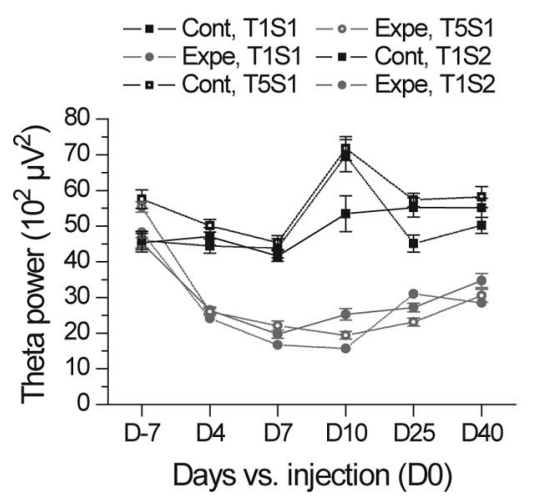

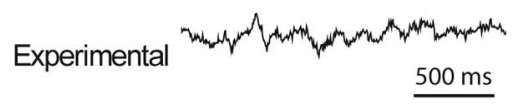

C During exploration

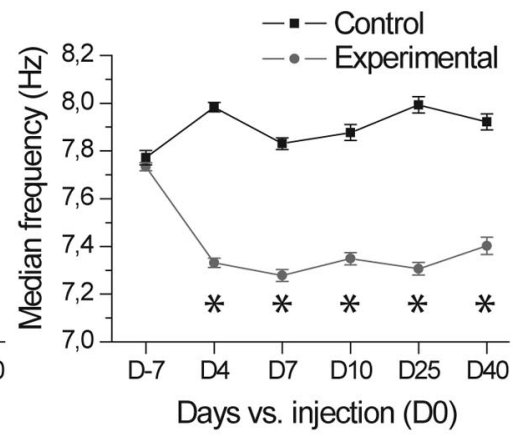

E

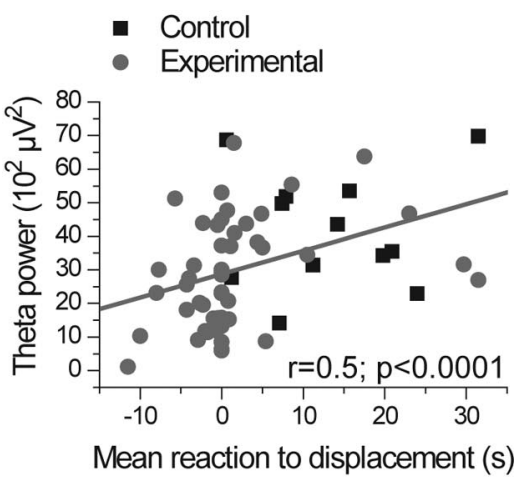

Figure 4. The amount of loss of theta activity positively correlates to spatial cognitive deficits in experimental animals $(n=$ 12). $A$, Examples of theta activity recorded during exploration in a control and in an experimental animal at D7. Note the decrease in amplitude and frequency in the experimental animal. $\boldsymbol{B}, \boldsymbol{C}$, Evolution of hippocampal theta $(4-12 \mathrm{~Hz})$ absolute power $(\boldsymbol{B})$ and mean frequency $(\boldsymbol{C})$ after $S E$ in control $(n=6)$ and experimental animals, during exploratory behavior $\left({ }^{*} p<0.0001\right)$. Note the stability of the recordings in controls and the early and significant $\left({ }^{*} p<0.0001\right)$ decrease in theta power and frequency in experimental animals. $\boldsymbol{D}$, Theta power is also decreased as soon as D4 during sessions 1 and 2 in experimental animals $\left({ }^{*} p<\right.$ 0.0001). $\boldsymbol{E}$, Spearman correlation between theta $(4-12 \mathrm{~Hz})$ absolute power during T1S2 and spatial memory performance evaluated as the difference of the time (s) spent exploring the displaced object during T1S2 and T5S1. Theta power correlates with spatial memory ( $r=0.5 ; p=0.00002$; experimental, $n=25$; control, $n=8)$. Data are expressed as means \pm SEM.

analysis showed a slight cell loss in all layers. However, the cell loss was a little more pronounced in layers I-II ( $8 \%$; pilo, 80784 \pm .1087 ; control, $88128 \pm 1517 ; p<0.05)$ than in layer III $(4 \%$; pilo, $114048 \pm 1746$; control, $119232 \pm 1389 ; p<0.05)$ and layers V-VI (3.6\%; pilo, $186624 \pm 2604$; control, $193536 \pm 1087$; $p<0.05$ ) (supplemental Table S5, available at www.jneurosci.org as supplemental material; Fig. $5 B$ ).

\section{Discussion}

Using a longitudinal study, we have demonstrated that experimental animals exhibit spatial memory deficits soon after the initial insult and that this deficit persists without further modification during epileptogenesis. In contrast, nonspatial memory does not seem affected. Our results reveal that the spatial memory deficit correlates with a decrease of the absolute theta power but less directly to ILA.

\section{Behavioral assessment}

The reaction-to-a-change paradigm used here allows us to test whether the animal has correctly encoded the spatial relation- ships between objects and their intrinsic (nonspatial) properties (Poucet et al., 1986). In both tests (object displacement and replacement), a comparison between the memory of the initial situation and the modified one is necessary. Hippocampal place cell disruption (Liu et al., 2003; Zhou et al., 2007; Lenck-Santini and Holmes, 2008), which would prevent adequate orientation of the animal in its environment, may negatively impact on spatial memory performance. Trace reactivation induces memory consolidation during immobility states or sleep (Chrobak and Buzsaki, 1994), a process which likely occurs in our behavioral paradigm during the 2 min of inter-trial rest. Spontaneous trace reactivation of the previous trial exploration may have not occurred in experimental animals. The deficit of habituation, which follows the same temporal profile, could be linked to similar functional alterations.

General hippocampal damage disrupts the reaction to a spatial change, leaving intact those to a new object (Morris, 1981; Aggleton et al., 1986; Save et al., 1992b). The spatial deficits found in epileptic animals (Rice et al., 1998; Hort et al., 1999; Dos Santos et al., 2005) could stem from cell loss and the reorganization of the underlying hippocampal structures (Morimoto et al., 2004; Engel et al., 2007), including inflammatory processes (Gobbo and O'Mara, 2004). The lack of reaction to a spatial change was not attributable to a nonspecific hypo-activity or to a general sick state in experimental animals, since interest to novelty was preserved. Our results extend previous data obtained in classical tasks such as the Morris Water Maze (Rice et al., 1998; Hort et al., 1999; Liu et al., 2003; Dos Santos et al., 2005), as the reaction-to-a-change task allows assessing both hippocampus-dependent spatial memory and nonhippocampus-dependent nonspatial memory in two consecutive tests.

The reorganization of hippocampal structures is an early phenomenon, starting soon after the initial SE, and continuing during epileptogenesis and the chronic period (Morimoto et al., 2004; El-Hassar et al., 2007). Cognitive deficits also appeared early after the initial insult and were not further modified during epileptogenesis. Network modifications that occur at later stages may be more relevant to the process of epileptogenesis and the formation of stable epileptogenic networks. Extra-limbic structures appear to sustain less damage (Morimoto et al., 2004), which may explain why nonspatial memory is not affected. Since we have only used a reaction to novelty paradigm, we cannot rule out that other nonspatial memories could be affected.

\section{Effect of ILA on cognitive processes}

In humans, cognitive activity can impact on ILA in a complex manner, as task difficulty influences epileptiform discharges (Kasteleijn-Nolst Trenité and Vermeiren, 2005). In the current 

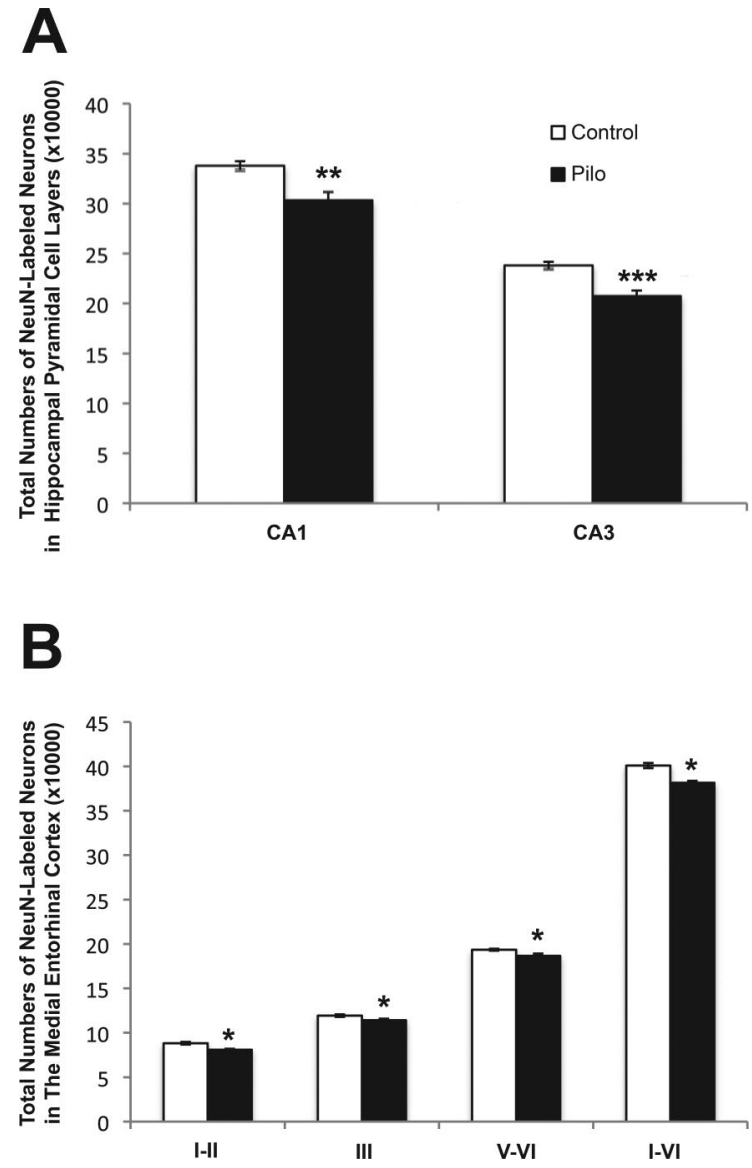

Figure 5. Slight cell loss of Neu-N-labeled neurons in the CA1-CA3 hippocampal pyramidal cell layers $(\boldsymbol{A})$ and all layers of the medial entorhinal cortex $(\boldsymbol{B}) .{ }^{*} p<0.05 ;{ }^{* *} p<0.01 ;{ }^{* * *} p<$ 0.005. Data are expressed as means \pm SEM.

study, however, there was no relationship between ILA and the memory tasks themselves, as all possible combinations were found (ILA at rest, which persisted during the task; ILA at rest, which stopped during the task; no ILA at rest, which started during the task) (supplemental Table S6, available at www. jneurosci.org as supplemental material). Thus, we found no correlation between spatial cognitive deficits and ILA measured before the task. However, we cannot rule out more complex correlations, such as the presence of ILA in structures connected to the CA1 region and not recorded here. Alternatively, ILA could have a negative impact when occurring during critical periods, e.g., trace reactivation (Chrobak and Buzsaki, 1994; Csicsvari et al., 2007) or place cell replay (Foster and Wilson, 2006; Molter et al., 2007). In epileptic patients, IA, unlike seizures, has no direct effect on cognition (Aldenkamp et al., 2001). However, IA has a small impact during "mechanistic" cognitive processes, such as attention or mental speed tasks (Aldenkamp et al., 2004).

\section{Theta rhythm}

Theta rhythm $(4-12 \mathrm{~Hz})$ is involved in many cognitive processes and, in particular, in spatial learning and memory (Hasselmo et al., 2002; Buzsaki, 2006). A loss of hippocampal theta rhythm results in spatial memory impairment in rats (Winson, 1978). Here, we found proportionality between cognitive performance for hippocampus-dependent tasks and theta power in the hippocampus. The largest amplitude theta in the hippocampus is observed in CA1 stratum lacunosum moleculare, where afferents from layer three entorhinal cortex synapse on pyramidal cell distal dendrites (Buzsaki, 2006). Although our recording electrodes were located close to stratum lacunosum moleculare (supplemental Fig. S4, available at www.jneurosci.org as supplemental material), their size did not allow the specific measurement of entorhinal cortex-driven theta. The recorded field rather reflects a global mean theta field in the CA1 region. Additional studies with multisite linear probes are necessary to determine the spatiotemporal evolution of theta dipoles during epileptogenesis.

Theta rhythm was significantly decreased in both power and frequency during exploratory behaviors in the open field, before and after the task. During the task, theta power was also decreased, but changes in theta median frequency could not be accurately measured because of a large variability between rats. Theta rhythm impairment may have also disturbed memory encoding and retrieval (Yamaguchi et al., 2004; Buzsaki, 2006). In addition to decreased theta power and frequency, a lack of coordination between theta generators/controllers may affect cognitive performance. In humans, unsuccessful memory formation during encoding is associated with a general decrease of rhinalhippocampal coherence and decreased theta oscillations (Fell et al., 2001, 2006). It will be important to determine whether synchrony is modified in different limbic structures. Alterations in theta oscillations followed the same temporal profile as spatial memory performance, further supporting the covariance of the two phenomena, with the reorganization of the underlying circuitry as a potential general mechanism. The loss of some entorhinal cortex neurons (Du et al., 1995; Kumar and Buckmaster, 2006), septal GABAergic cells (Garrido Sanabria et al., 2006), CA1 oriens-lacunosum moleculare GABAergic interneurons (Cossart et al., 2001; Dinocourt et al., 2003; Dugladze et al., 2007), altered theta amplification mechanisms (Marcelin et al., 2009), as well as inflammatory processes (Gobbo and O'Mara, 2004), early during epileptogenesis may play a direct role in theta impairment, since these cell types are strongly involved in theta generation/control.

\section{Validity of the model}

Pilocarpine injection triggers an SE, followed by a seizure-free latent period, before the occurrence of spontaneous recurrent seizures. The analysis of temporal pole networks reveals considerable modifications, which evolve in time, eventually leading to hippocampal sclerosis (Morimoto et al., 2004; El-Hassar et al., 2007). A similar remodeling has been found in patients with TLE (Du et al., 1993; Plate et al., 1993; Mathern et al., 1996). The alterations in spatial memory and theta rhythm we describe may be a direct consequence of the initial injury resulting from the status epilepticus (cell loss, transcriptional, translational, and post-translational modification, etc.). The extent of the reorganization soon after SE may explain why cognitive function and theta oscillations are not further altered when animals have spontaneous seizures. In contrast, in the kindling model of TLE where there is no SE, the cognitive decline parallels the number of seizures (Kotloski et al., 2002). This suggests that the time course of cognitive and theta dysfunctions depend upon the initial condition leading to epilepsy. Whether the results we describe are relevant to post-SE modifications and/or epileptogenesis in human remains an open question. However, they provide working hypotheses that can be tested in at-risk patients.

\section{Conclusion}

Cognitive functions and pathological neural activities (ILA and seizures) share the same underlying circuitry. This circuitry is 
considerably remodeled after SE, many parameters undergoing modification with specific temporal profiles. Some of these alterations may contribute to both cognitive deficits and pathological activities, whereas others may be specific. Network remodeling occurs on a very fast time scale after SE, leading to rapid disruption of theta activity and spatial memory impairment. These modifications, together with the occurrence of ILA, may constitute early markers of epilepsy. It remains to be determined whether theta activity and spatial memory deficits are dissociated from epileptogenesis per se, i.e., whether they are a direct consequence of network remodeling. Interestingly, memory deficits and morpho-functional reorganization of the hippocampus occur in other neurological pathologies, e.g., Huntington's disease (Kohl et al., 2007) and Alzheimer's disease (Allen et al., 2007). The alterations we describe may bear a more general value, outside the epilepsy field.

\section{References}

Aggleton JP, Hunt PR, Rawlins JN (1986) The effects of hippocampal lesions upon spatial and non-spatial tests of working memory. Behav Brain Res 19:133-146.

Aldenkamp A, Arends J (2004) The relative influence of epileptic EEG discharges, short nonconvulsive seizures, and type of epilepsy on cognitive function. Epilepsia 45:54-63.

Aldenkamp AP, Arends J, Overweg-Plandsoen TC, van Bronswijk KC, Schyns-Soeterboek A, Linden I, Diepman L (2001) Acute cognitive effects of nonconvulsive difficult-to-detect epileptic seizures and epileptiform electroencephalographic discharges. J Child Neurol 16:119-123.

Aldenkamp AP, Arends J, Verspeek S, Berting M (2004) The cognitive impact of epileptiform EEG-discharges; relationship with type of cognitive task. Child Neuropsychol 10:297-305.

Allen G, Barnard H, McColl R, Hester AL, Fields JA, Weiner MF, Ringe WK, Lipton AM, Brooker M, McDonald E, Rubin CD, Cullum CM (2007) Reduced hippocampal functional connectivity in Alzheimer disease. Arch Neurol 64:1482-1487.

Berlyne D (1950) Novelty and curiosity as determinants of exploratory behaviour. Br J Psychol 41:68-80.

Boulland JL, Ferhat L, Tallak Solbu T, Ferrand N, Chaudhry FA, StormMathisen J, Esclapez M (2007) Changes in vesicular transporters for gamma-aminobutyric acid and glutamate reveal vulnerability and reorganization of hippocampal neurons following pilocarpine-induced seizures. J Comp Neurol 503:466-485.

Butler CR, Zeman AZ (2008) Recent insights into the impairment of memory in epilepsy: transient epileptic amnesia, accelerated long-term forgetting and remote memory impairment. Brain 131:2243-2263.

Buzsaki G, ed (2006) Rhythms of the brain. New York: Oxford UP.

Chrobak JJ, Buzsáki G (1994) Selective activation of deep layer (V-VI) retrohippocampal cortical neurons during hippocampal sharp waves in the behaving rat. J Neurosci 14:6160-6170.

Clifford DB, Olney JW, Maniotis A, Collins RC, Zorumski CF (1987) The functional anatomy and pathology of lithium-pilocarpine and high-dose pilocarpine seizures. Neuroscience 23:953-968.

Cornaggia CM, Beghi M, Provenzi M, Beghi E (2006) Correlation between cognition and behavior in epilepsy. Epilepsia 47 [Suppl]2:34-39.

Cossart R, Dinocourt C, Hirsch JC, Merchan-Perez A, De Felipe J, Ben-Ari Y, Esclapez M, Bernard C (2001) Dendritic but not somatic GABAergic inhibition is decreased in experimental epilepsy. Nat Neurosci 4:52-62.

Csicsvari J, O’Neill J, Allen K, Senior T (2007) Place-selective firing contributes to the reverse-order reactivation of CA1 pyramidal cells during sharp waves in open-field exploration. Eur J Neurosci 26:704-716.

Dinocourt C, Petanjek Z, Freund TF, Ben-Ari Y, Esclapez M (2003) Loss of interneurons innervating pyramidal cell dendrites and axon initial segments in the CA1 region of the hippocampus following pilocarpineinduced seizures. J Comp Neurol 459:407-425.

Dos Santos JG Jr, Longo BM, Blanco MM, Menezes de Oliveira MG, Mello LE (2005) Behavioral changes resulting from the administration of cycloheximide in the pilocarpine model of epilepsy. Brain Res 1066:37-48.

Du F, Whetsell WO Jr, Abou-Khalil B, Blumenkopf B, Lothman EW, Schwarcz R (1993) Preferential neuronal loss in layer III of the entorhi- nal cortex in patients with temporal lobe epilepsy. Epilepsy Res 16:223-233.

Du F, Eid T, Lothman EW, Köhler C, Schwarcz R (1995) Preferential neuronal loss in layer III of the medial entorhinal cortex in rat models of temporal lobe epilepsy. J Neurosci 15:6301-6313.

Dugladze T, Vida I, Tort AB, Gross A, Otahal J, Heinemann U, Kopell NJ, Gloveli T (2007) Impaired hippocampal rhythmogenesis in a mouse model of mesial temporal lobe epilepsy. Proc Natl Acad Sci U S A 104:17530-17535.

El-Hassar L, Milh M, Wendling F, Ferrand N, Esclapez M, Bernard C (2007) Cell domain-dependent changes in the glutamatergic and GABAergic drives during epileptogenesis in the rat CAl region. J Physiol 578:193-211.

Engel J Jr, Pedley TA, Aicardi J, Dichter MA, Moshé S, eds (2007) Epilepsy. A comprehensive textbook, Ed 2. Philadelphia: Lippincott Williams \& Wilkins.

Esclapez M, Hirsch JC, Ben-Ari Y, Bernard C (1999) Newly formed excitatory pathways provide a substrate for hyperexcitability in experimental temporal lobe epilepsy. J Comp Neurol 408:449-460.

Fell J, Klaver P, Lehnertz K, Grunwald T, Schaller C, Elger CE, Fernández G (2001) Human memory formation is accompanied by rhinalhippocampal coupling and decoupling. Nat Neurosci 4:1259-1264.

Fell J, Fernández G, Lutz MT, Kockelmann E, Burr W, Schaller C, Elger CE, Helmstaedter C (2006) Rhinal-hippocampal connectivity determines memory formation during sleep. Brain 129:108-114.

Foster DJ, Wilson MA (2006) Reverse replay of behavioural sequences in hippocampal place cells during the awake state. Nature 440:680-683.

Garrido Sanabria ER, Castañeda MT, Banuelos C, Perez-Cordova MG, Hernandez S, Colom LV (2006) Septal GABAergic neurons are selectively vulnerable to pilocarpine-induced status epilepticus and chronic spontaneous seizures. Neuroscience 142:871-883.

Gobbo OL, O'Mara SM (2004) Post-treatment, but not pre-treatment, with the selective cyclooxygenase-2 inhibitor celecoxib markedly enhances functional recovery from kainic acid-induced neurodegeneration. Neuroscience 125:317-327.

Hasselmo ME, Hay J, Ilyn M, Gorchetchnikov A (2002) Neuromodulation, theta rhythm and rat spatial navigation. Neural Netw 15:689-707.

Herman ST (2002) Epilepsy after brain insult: targeting epileptogenesis. Neurology 59:S21-S26.

Holmes GL, Lenck-Santini PP (2006) Role of interictal epileptiform abnormalities in cognitive impairment. Epilepsy Behav 8:504-515.

Hort J, Broźek G, Mares P, Langmeier M, Komárek V (1999) Cognitive functions after pilocarpine-induced status epilepticus: changes during silent period precede appearance of spontaneous recurrent seizures. Epilepsia 40:1177-1183.

Kahana MJ, Sekuler R, Caplan JB, Kirschen M, Madsen JR (1999) Human theta oscillations exhibit task dependence during virtual maze navigation. Nature 399:781-784.

Kasteleijn-Nolst Trenité DG, Vermeiren R (2005) The impact of subclinical epileptiform discharges on complex tasks and cognition: relevance for aircrew and air traffic controllers. Epilepsy Behav 6:31-34.

Kinney GG, Patino P, Mermet-Bouvier Y, Starrett JE Jr, Gribkoff VK (1999) Cognition-enhancing drugs increase stimulated hippocampal theta rhythm amplitude in the urethane-anesthetized rat. J Pharmacol Exp Ther 291:99-106.

Kohl Z, Kandasamy M, Winner B, Aigner R, Gross C, Couillard-Despres S, Bogdahn U, Aigner L, Winkler J (2007) Physical activity fails to rescue hippocampal neurogenesis deficits in the R6/2 mouse model of Huntington's disease. Brain Res 1155:24-33.

Kotloski R, Lynch M, Lauersdorf S, Sutula T (2002) Repeated brief seizures induce progressive hippocampal neuron loss and memory deficits. Prog Brain Res 135:95-110.

Kumar SS, Buckmaster PS (2006) Hyperexcitability, interneurons, and loss of GABAergic synapses in entorhinal cortex in a model of temporal lobe epilepsy. J Neurosci 26:4613-4623.

Lenck-Santini PP, Holmes GL (2008) Altered phase precession and compression of temporal sequences by place cells in epileptic rats. J Neurosci 28:5053-5062.

Liu X, Muller RU, Huang LT, Kubie JL, Rotenberg A, Rivard B, Cilio MR, Holmes GL (2003) Seizure-induced changes in place cell physiology: relationship to spatial memory. J Neurosci 23:11505-11515.

Liu Z, Nagao T, Desjardins GC, Gloor P, Avoli M (1994) Quantitative eval- 
uation of neuronal loss in the dorsal hippocampus in rats with long-term pilocarpine seizures. Epilepsy Res 17:237-247.

Lopes da Silva FH, Gorter JA, Wadman WJ (1986) Kindling of the hippocampus induces spatial memory deficits in the rat. Neurosci Lett 63:115-120.

Majak K, Pitkanen A (2004) Do seizures cause irreversible cognitive damage? Evidence from animal studies. Epilepsy Behav 5 [Suppl]1:S35-S44.

Marcelin B, Chauvière L, Becker A, Migliore M, Esclapez M, Bernard C (2009) h channel-dependent deficit of theta oscillation resonance and phase shift in temporal lobe epilepsy. Neurobiol Dis 33:436-447.

Marques CM, Caboclo LO, da Silva TI, Noffs MH, Carrete H Jr, Lin K, Lin J, Sakamoto AC, Yacubian EM (2007) Cognitive decline in temporal lobe epilepsy due to unilateral hippocampal sclerosis. Epilepsy Behav 10:477-485.

Mathern GW, Babb TL, Leite JP, Pretorius K, Yeoman KM, Kuhlman PA (1996) The pathogenic and progressive features of chronic human hippocampal epilepsy. Epilepsy Res 26:151-161.

Maurer AP, Vanrhoads SR, Sutherland GR, Lipa P, McNaughton BL (2005) Self-motion and the origin of differential spatial scaling along the septotemporal axis of the hippocampus. Hippocampus 15:841-852.

McNaughton N, Ruan M, Woodnorth MA (2006) Restoring theta-like rhythmicity in rats restores initial learning in the Morris water maze. Hippocampus 16:1102-1110.

Meador KJ (2006) Cognitive and memory effects of the new antiepileptic drugs. Epilepsy Res 68:63-67.

Meador KJ (2007) The basic science of memory as it applies to epilepsy. Epilepsia 48 [Suppl]9:23-25.

Molter C, Sato N, Yamaguchi Y (2007) Reactivation of behavioral activity during sharp waves: a computational model for two stage hippocampal dynamics. Hippocampus 17:201-209.

Morimoto K, Fahnestock M, Racine RJ (2004) Kindling and status epilepticus models of epilepsy: rewiring the brain. Prog Neurobiol 73:1-60.

Morris RG (1981) Spatial localization does not require the presence of local cues. Learn Motiv 12:239-260.

Mulders WH, West MJ, Slomianka L (1997) Neuron numbers in the presubiculum, parasubiculum, and entorhinal area of the rat. J Comp Neurol 385:83-94.

Nolan MF, Malleret G, Dudman JT, Buhl DL, Santoro B, Gibbs E, Vronskaya S, Buzsáki G, Siegelbaum SA, Kandel ER, Morozov A (2004) A behavioral role for dendritic integration: HCN1 channels constrain spatial memory and plasticity at inputs to distal dendrites of CA1 pyramidal neurons. Cell 119:719-732.
Obenaus A, Esclapez M, Houser CR (1993) Loss of glutamate decarboxylase mRNA-containing neurons in the rat dentate gyrus following pilocarpine-induced seizures. J Neurosci 13:4470-4485.

Paxinos G, Watson C (2005) The rat brain in stereotaxic coordinates, Ed 5. Burlington, MA: Academic.

Plate KH, Wieser HG, Yasargil MG, Wiestler OD (1993) Neuropathological findings in 224 patients with temporal lobe epilepsy. Acta Neuropathol 86:433-438.

Poucet B, Durup M, Thinus-Blanc C (1986) A study of exploratory behaviour as an index of spatial knowledge in hamsters. Anim Learn Behav 14:93-100.

Rice AC, Floyd CL, Lyeth BG, Hamm RJ, DeLorenzo RJ (1998) Status epilepticus causes long-term NMDA receptor-dependent behavioral changes and cognitive deficits. Epilepsia 39:1148-1157.

Save E, Poucet B, Foreman N, Buhot MC (1992a) Object exploration and reactions to spatial and nonspatial changes in hooded rats following damage to parietal cortex or hippocampal formation. Behav Neurosci 106:447-456.

Save E, Buhot MC, Foreman N, Thinus-Blanc C (1992b) Exploratory activity and response to a spatial change in rats with hippocampal or posterior parietal cortical lesions. Behav Brain Res 47:113-127.

Staley K, Hellier JL, Dudek FE (2005) Do interictal spikes drive epileptogenesis? Neuroscientist 11:272-276.

Thinus-Blanc C, ed (1996) Animal spatial cognition. Behavioral and neural approaches. Singapore: World Scientific.

West MJ (1999) Stereological methods for estimating the total number of neurons and synapses: issues of precision and bias. Trends Neurosci 22:51-61.

West MJ, Slomianka L, Gundersen HJ (1991) Unbiased stereological estimation of the total number of neurons in thesubdivisions of the rat hippocampus using the optical fractionator. Anat Rec 231:482-497.

White HS (2002) Animal models of epileptogenesis. Neurology 59:S7-S14.

Winson J (1978) Loss of hippocampal theta rhythm results in spatial memory deficit in the rat. Science 201:160-163.

Yamaguchi Y, Aota Y, Sato N, Wagatsuma H, Wu Z (2004) Synchronization of neural oscillations as a possible mechanism underlying episodic memory: a study of theta rhythm in the hippocampus. J Integr Neurosci 3:143-157.

Zhou JL, Lenck-Santini PP, Zhao Q, Holmes GL (2007) Effect of interictal spikes on single-cell firing patterns in the hippocampus. Epilepsia 48: $720-731$. 